\title{
Cardiorenal Protection in Diabetic Kidney Disease
}

\author{
Jason F. Lee ${ }^{1,2, *}$, Ecaterina Berzan ${ }^{1,2, *}$, Vikas S. Sridhar ${ }^{1,2,3, *}$, Ayodele Odutayo ${ }^{2,4}$, David Z.I. Cherney , $^{1,2,3,5,6}$ \\ ${ }^{1}$ Toronto General Hospital Research Institute, University Health Network; ${ }^{2}$ Division of Nephrology, Department of Medicine, \\ University of Toronto; ${ }^{3}$ Banting and Best Diabetes Centre; ${ }^{4}$ Applied Health Research Centre, Li Ka Shing Knowledge Institute of \\ St Michael's Hospital; Departments of ${ }^{5}$ Physiology, ${ }^{6}$ Pharmacology and Toxicology, University of Toronto, Toronto, ON, Canada
}

Over the last 5 years there have been many new developments in the management of diabetic kidney disease. Glucagon-like peptide-1 receptor agonists (GLP-1 RA) and sodium-glucose cotransporter-2 (SGLT2) inhibitors were initially used for glycemic control, but more recent studies have now shown that their benefits extend to cardiovascular and kidney outcomes. The recent addition of data on the novel mineralocorticoid receptor antagonist (MRA) gives us another approach to further decrease the residual risk of diabetic kidney disease progression. In this review we describe the mechanism of action, key studies, and possible adverse effects related to these three classes of medications. The management of type 2 diabetes now includes an increasing number of medications for the management of comorbidities in a patient population at significant risk of cardiovascular disease and progression of chronic kidney disease. It is from this perspective that we seek to outline the rationale for the sequential and/or combined use of SGLT2 inhibitors, GLP-1 RA and MRAs in patients with type 2 diabetes for heart and kidney protection.

Keywords: Diabetes mellitus, type 2; Diabetic nephropathies; Heart failure; Cardiovascular diseases; Glucagon-like peptide-1 receptor; Mineralocorticoid receptor antagonists; Sodium-glucose transporter 2 inhibitors; Renal insufficiency, chronic

\section{INTRODUCTION}

Until recently, glycemic control, blood pressure management, angiotensin converting enzyme (ACE) inhibitors or angiotensin receptor blockers (ARB) were the only available strategies to prevent diabetic kidney disease (DKD) progression. Older glucose lowering therapies demonstrated efficacy with respect to achieving glycemic targets but lacked benefits for 'hard' kidney or cardiovascular (CV) outcomes [1]. Importantly, older glucose lowering therapies were also associated with potential harm related to heart failure (HF) hospitalizations with thiazolidinediones, as well as hypoglycemia and weight gain with therapies such as sulfonylureas and insulin [2].
Over the last 5 years, with the emergence of newer agents including sodium-glucose cotransporter-2 (SGLT2) inhibitors, glucagon-like peptide-1 receptor agonists (GLP-1 RA) and novel mineralocorticoid receptor antagonist (MRA), management of type 2 diabetes mellitus (T2DM) and DKD has since shifted away from a 'glucose-centric' focus towards one of 'heart and kidney protection [3-5]. These newer agents have individually demonstrated $\mathrm{CV}$ and/or kidney benefits, and the combined use of these agents is particularly appealing due to potential mechanistic and clinical synergies [6]. Accordingly, our aim was to review the mechanisms and clinical benefits of these three classes of agents and outline the case for their combined use for heart and kidney protection in DKD.
Received: 5 February 2021, Revised: 17 March 2021, Accepted: 26 March 2021

Corresponding author: David Z.I. Cherney

Toronto General Hospital Research Institute, University Health Network, 585 University Ave, 8N-845, Toronto, ON M5G 2N2, Canada

Tel: +1-416-340-4151, Fax: +1-416-340-4999, E-mail: david.cherney@uhn.ca

\section{Copyright $\odot 2021$ Korean Endocrine Society}

This is an Open Access article distributed under the terms of the Creative Commons Attribution Non-Commercial License (https://creativecommons.org/ licenses/by-nc/4.0/) which permits unrestricted non-commercial use, distribution, and reproduction in any medium, provided the original work is properly cited.

*These authors contributed equally to this work. 
SGLT2 INHIBITORS, CARDIOVASCULAR AND KIDNEY PROTECTION IN PATIENTS WITH T2DM

\section{SGLT2 inhibition and mechanistic benefits}

The precise mechanisms behind heart and kidney benefits of SGLT2 inhibitors are incompletely understood, though there are likely multiple complementary physiological effects $[7,8]$. Kidney protective mechanisms of SGLT2 inhibitors are commonly considered according to hemodynamic versus non-hemodynamic actions. By inhibiting proximal tubular sodium and glucose reabsorption, SGLT2 inhibitors increase distal sodium delivery to the macula densa and restore tubuloglomerular feedback, with the effect of decreasing intraglomerular pressure, shear stress, hyperfiltration and albuminuria [9-12]. The cardio-protective mechanisms linked with SGLT2 inhibitors also overlap with those associated with kidney protection. For example, beyond restoring glomerular pressure toward more normal physiological levels, hemodynamic effects in the systemic circulation induced by natriuresis restore relative euvolemia, lower blood pressure and arterial stiffness, and may contribute to improving cardiac preload and afterload $[13,14]$.

Independent of hemodynamic effects, from a metabolic perspective, SGLT2 inhibitors promote glucosuria and modestly improve glycemic control $[15,16]$. The associated caloric loss also contributes to reductions in body weight by 2 to $3 \mathrm{~kg}$ on average, another traditional CV risk factor [15,17]. Glucosuria also enhances gluconeogenesis and ketogenesis, part of an SGLT2 inhibitor induced shift to a 'fasting state.' Ketogenesis is hypothesized to be a direct mediator of cardioprotection with SGLT2 inhibition since ketone bodies are an efficient energy source with the potential to improve myocardial energy dynamics and exert direct anti-inflammatory effects [18-20]. Alternatively, ketones may simply be an indirect biomarker of heart and kidney protection, with SGLT2 inhibitors activating 'nutrientdeprivation pathways,' leading to improved mitochondrial function and energy dynamics and less oxidative stress at the cellular level, with ketogenesis simply being a byproduct of this process [21-23]. These metabolic changes activated by 'fasting state' with SGLT2 inhibition may also ameliorate myocardial efficiency and hypoxia. SGLT2 inhibitors also inhibit myocardial sodium-hydrogen exchange, with hypothesized downstream benefits to cardiac hypertrophy and fibrosis [13]. SGLT2 inhibitors may also reduce energy requirements and hypoxia within proximal tubular cells, and may improve oxygen delivery by increasing hematocrit [24]. This, combined with their modulation of neurohumoral pathways and reduction of glucose entry into tubular cells, likely contributes to suppression of kidney hypoxia, inflammation, and fibrosis [25-29]. Finally, improvements in kidney function itself may indirectly improve cardiac function through reductions in sympathetic nervous system activation, systemic inflammation and chronic hypervolemia that characterize the chronic cardiorenal syndrome [30].

\section{SGLT2 inhibition and clinical heart and kidney protection}

The heart and kidney protective effects of SGLT2 inhibitors were first demonstrated in three large cardiovascular outcome trials (CVOT) exclusively comprising participants with T2DM. The Empagliflozin Cardiovascular Outcome Event Trial in Type 2 Diabetes Mellitus Patients (EMPA-REG OUTCOME), Canagliflozin Cardiovascular Assessment Study (CANVAS Program) and Multicenter Trial to Evaluate the Effect of Dapagliflozin on the Incidence of Cardiovascular Events-Thrombolysis in Myocardial Infarction 58 (DECLARE-TIMI-58) trials included $>34,000$ patients and demonstrated significant reductions in the risk of major adverse cardiovascular events (MACE; composite of non-fatal stroke, non-fatal myocardial infarction and $\mathrm{CV}$ death) and/or hospitalization for heart failure (HHF) (Table 1 for a summary of effects) [31-33].

Secondary kidney composite outcomes were also consistently reduced across the three trials. In EMPA-REG OUTCOME, the kidney composite of albuminuria progression, doubling of serum creatinine, end-stage kidney disease (ESKD), or renal death was reduced by $39 \%$, with consistent benefits across multiple subgroups including those with chronic kidney disease (CKD) at baseline [34]. In the CANVAS Program, the kidney composite (doubling of serum creatinine, renal replacement therapy or renal death) was similarly reduced by $47 \%$ (hazard ratio [HR], 0.53 ; $95 \%$ confidence interval [CI], 0.33 to 0.84 ) [35]. By design, DECLARE-TIMI-58 had the lowest CV risk cohort and the highest baseline mean estimated glomerular filtration rate (eGFR) of the three trials [32]. In this lower kidney risk cohort, dapagliflozin reduced the composite kidney endpoint, which included a more modest $40 \%$ decline in eGFR, ESKD or renal death, by $47 \%$ (HR, 0.53 ; 95\% CI, 0.43 to 0.66 ) [13]. Risk reduction for $\mathrm{CV}$ outcomes was similar across baseline levels of kidney risk, aside from a lower HR in the impaired eGFR with albuminuria group [36]. Post hoc analyses have demonstrated that observed CV and kidney benefits in the original CVOTs were largely independent of glycemic lowering [37-40].

Two subsequent CVOTs were published in 2020. Cardiovascular Outcomes Following Ertugliflozin Treatment in Type 2 


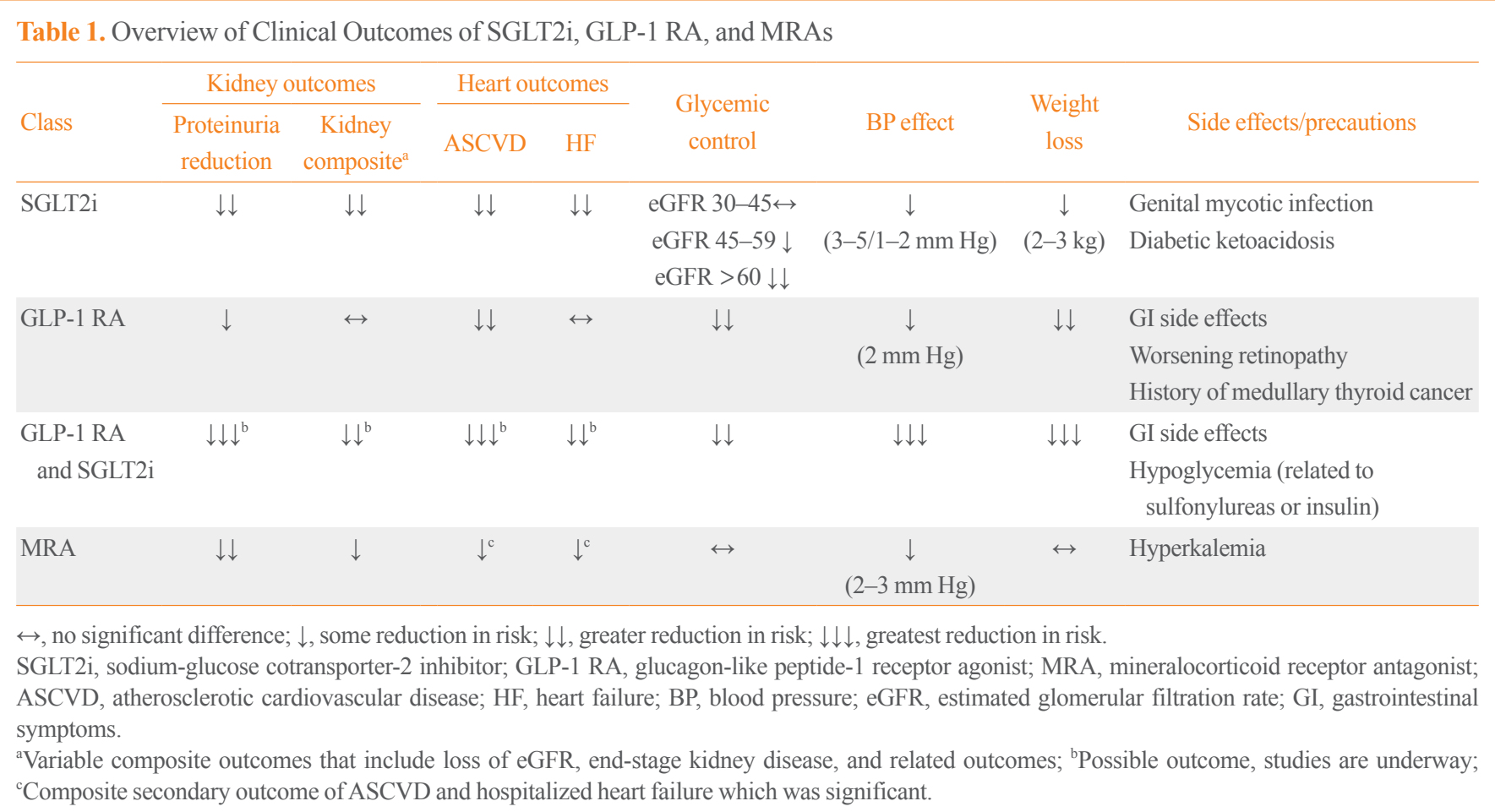

Diabetes Mellitus Participants with Vascular Disease (VERTISCV), which compared ertugliflozin with placebo showed noninferiority in regards to its primary MACE outcome, as well as a 30\% reduction in HHF [41]. For the key composite kidney endpoint, which used "doubling of serum creatinine" for the definition of significant kidney function loss, the impact of ertugliflozin was neutral [41]. However, as presented at the European Association for the Study of Diabetes 2020, with a more modest kidney endpoint comprised of a sustained $40 \%$ eGFR decline, a HR of 0.66 was observed ( $95 \%$ CI, 0.50 to 0.88 ) [42]. Additionally, when stratified by kidney risk in VERTIS-CV as determined by KDIGO risk categories, significant reductions in the HHF/CV death composite were observed in the moderate and high/very high risk categories ( $P=0.03$ for interaction) [43].

In a second trial, Effect of Sotagliflozin on Cardiovascular and Renal Events in Patients With Type 2 Diabetes and Moderate Renal Impairment Who Are at Cardiovascular Risk (SCORED), a dual SGLT2 inhibitor and gastrointestinal SGLT1 inhibitor sotagliflozin was examined in patients with T2DM, CKD (eGFR from 25 to $60 \mathrm{~mL} / \mathrm{min} / 1.73 \mathrm{~m}^{2}$ ) and CV risk factors. Although the trial was stopped early due to a lack of funding, the trial investigators demonstrated a reduction in the primary cardiac composite endpoint (total number of deaths from CV causes, HHF and urgent visits for HF) of $26 \%$ (HR, 0.74 ;
$95 \%$ CI, 0.63 to 0.88 ) [44]. While the composite kidney outcome including a $50 \%$ sustained decrease in eGFR was not statistically significant (HR, $0.71 ; 95 \% \mathrm{CI}, 0.46$ to 1.08 ), analyses with other kidney composites have not yet been published. Sotagliflozin also demonstrated safety and efficacy in patients with worsening HF in Effect of Sotagliflozin on Cardiovascular Events in Patients With Type 2 Diabetes Post Worsening Heart Failure (SOLOIST-WHF), although this trial was also stopped early due to insufficient funding [45].

Analyses of kidney outcomes in the original CVOTs prompted dedicated kidney outcome trials, of which two are completed: Evaluation of the Effects of Canagliflozin on Renal and Cardiovascular Outcomes in Participants With Diabetic Nephropathy (CREDENCE, NCT02065791) and A Study to Evaluate the Effect of Dapagliflozin on Renal Outcomes and Cardiovascular Mortality in Patients With Chronic Kidney Disease (DAPA-CKD, NCT03036150). These trials were both stopped early due to clinical benefit. CREDENCE included patients with an eGFR from 30 to $90 \mathrm{~mL} / \mathrm{min} / 1.73 \mathrm{~m}^{2}$ and a urine albumin-to-creatinine ratio (UACR) of 300 to $5,000 \mathrm{mg} / \mathrm{g}$. The kidney specific outcome was a composite of ESKD, doubling of creatinine or death from a renal cause, and was reduced by $34 \%$ in the treatment group (HR, $0.66 ; 95 \% \mathrm{CI}, 0.53$ to 0.81 ) [46]. DAPA-CKD had broader inclusion criteria, dropping the eGFR 
and UACR cutoffs and including participants without diabetes. This trial enrolled 4,304 participants with an eGFR of 25 to 75 $\mathrm{mL} / \mathrm{min} / 1.73 \mathrm{~m}^{2}$ with a UACR of 200 to $5,000 \mathrm{mg} / \mathrm{g}$. The primary outcome was a composite of a sustained decline in eGFR of at least $50 \%$, ESKD, or death from renal or CV causes. Dapagliflozin reduced the risk of the primary outcome by $44 \%$ (HR, $0.56 ; 95 \%$ CI, 0.45 to 0.68 ) with a number needed to treat of 19 and an overall mortality benefit (HR, $0.69 ; 95 \% \mathrm{CI}, 0.53$ to 0.88). Importantly, $32.5 \%$ of patients enrolled did not have T2DM and $14.5 \%$ had an eGFR below $30 \mathrm{~mL} / \mathrm{min} / 1.73 \mathrm{~m}^{2}$, demonstrating the benefit of dapagliflozin across a range of kidney function regardless of diabetes status [47]. Please see Table 1 which summarizes the key findings. In addition to these completed trials, The Study of Heart and Kidney Protection With Empagliflozin (EMPA-KIDNEY, NCT03594110) is currently underway and is recruiting a broader group of participants with eGFRs as low as $20 \mathrm{~mL} / \mathrm{min} / 1.73 \mathrm{~m}^{2}$, without the requirement for albuminuria in patients with lower eGFR between 20 to 45 $\mathrm{mL} / \mathrm{min} / 1.73 \mathrm{~m}^{2}$. Therefore, this innovative and important trial design feature has the potential to extend the use of this class of medication to those with normo- or microalbuminuria - a subgroup of patients who have yet to be included in prior key kidney protection trials.

Beyond clinical kidney benefits, CVOT and kidney outcome trials have consistently demonstrated benefits on HF outcomes, leading to dedicated HF outcome trials. The Study to Evaluate the Effect of Dapagliflozin on the Incidence of Worsening Heart Failure or Cardiovascular Death in Patients With Chronic Heart Failure (DAPA-HF, NCT03036124) and Empagliflozin Outcome Trial in Patients With Chronic Heart Failure With Reduced Ejection Fraction (EMPEROR Reduced, NCT03057977) both demonstrated HF [48,49] and kidney benefits [50,51] in participants with HF and reduced ejection fraction with and without diabetes. Results of the Dapagliflozin Evaluation to Improve the Lives of Patients With Preserved Ejection Fraction Heart Failure (DELIVER, NCT03619213) and Empagliflozin Outcome Trial in Patients With Chronic Heart Failure With Preserved Ejection Fraction (EMPEROR Preserved, NCT03057951) in participants with HF and preserved ejection fraction are awaited.

\section{SGLT2 inhibition and safety}

Overall, clinical trials have reported lower rates of total adverse events with SGLT2 inhibition $[32,33,46]$. A secondary analysis of CREDENCE showed no differences in the rate of adverse events when stratified by GFR [52]. Mycotic genital infections remain among the most common side effects while diabetic ketoacidosis is an uncommon but serious complication [53]. An increased risk of amputations was reported in the CANVAS Program trial but has not been demonstrated in any other clinical trial nor was there any signal of this issue in a meta-analysis involving 57,713 participants [54]. Similarly, despite theoretical concerns around an increased risk of acute kidney injury (AKI), SGLT2 inhibitors have been associated with lower rates of AKI, possibly due to prevention of ischemia in the kidney $[55,56]$.

\section{GLP-1 RAs, CARDIOVASCULAR AND RENAL PROTECTION IN PATIENTS WITH T2DM}

\section{GLP-1 RA and effects on metabolism}

GLP-1 RAs increase insulin secretion, stimulate glucose uptake and delay gastric emptying, leading to significant reductions in glycated hemoglobin (HbA1c) and a greater likelihood of achieving an HbA1c of $\leq 7 \%$ [57]. GLP-1 RAs also increase self-reported post-prandial satiety, thereby reducing energy intake by $16 \%$ [58] and body weight by 2 to $4 \mathrm{~kg}$ [57]. Weight loss stems primarily from a reduction in body fat as opposed to lean mass and this weight change is sustained over long-term follow-up [59]. The mechanisms responsible for weight loss are in part due to GLP-1 RA effects on appetite and satiety control centers in the central nervous system [60].

\section{GLP-1 RA and effects on natriuresis, blood pressure, and kidney function}

Although GLP-1 receptors are found in CV tissues [61], the effect of GLP-1 RAs on CV physiological parameters such as blood pressure and heart rate is modest. In randomized controlled trials, GLP-1 RAs reduce systolic blood pressure by approximately 1 to $2 \mathrm{~mm} \mathrm{Hg}$, with lesser effects on diastolic blood pressure [62]. In addition, GLP-1 RAs did not reduce the incidence of new onset hypertension compared to placebo [62] and increased heart rate by 2 to 3 beats per minute compared to placebo [62]. Based on these modest effects, it is not clear if blood pressure lowering with GLP-1 RAs contributes mechanistically for improved cardiorenal outcomes with these agents in pivotal clinical trials (discussed below).

Beyond effects on blood pressure, glucose homeostasis and metabolism, GLP-1 RAs have additional effects on the kidney and CV system (see Table 1 for a summary of effects). In the kidney, GLP-1 receptors are located at the afferent arteriole [63] and proximal tubule [64], which leads to divergent acute effects 
on renal hemodynamics. For instance, in a non-randomized crossover study of healthy obese men who were administered exenatide, there was a $33 \%$ reduction in afferent arteriolar resistance and no change in efferent arteriolar resistance compared to placebo after a single dose [65]. The net effects were increases in renal blood flow and glomerular filtration rate [65]. Of interest, these renal hemodynamic changes were attenuated by concomitant administration of exenatide and the nitric oxide (NO)-synthase inhibitor L-NG-monomethyl arginine [65], a finding which has been confirmed in subsequent experimental studies [66]. These observations suggest that the effects of GLP1 RAs at the afferent arteriole is in part mediated by NO.

In addition to effects on NO, GLP-1 RAs may impact other vasoactive mediators such as the renin-angiotensin-aldosterone system (RAAS). In a crossover study of healthy men who received a 2-hour infusion of synthetic human GLP-1, there was a $19 \%$ decrease in circulating angiotensin II levels, but no change in plasma levels of renin or aldosterone [67], and no alteration in renal hemodynamic function [67]. This decrease in circulating markers of the RAAS has not been consistently observed in human mechanistic studies, possibly due to differences in the study cohorts involved in these trials [68]. Further work is required to elucidate the relationship between GLP-1 RAs and neurohormonal activation, including the RAAS, and whether there are any implications for renal or systemic hemodynamic function, or clinical endpoints.

Through interactions with GLP-1 receptors in the proximal tubules, GLP-1 RAs also have natriuretic and diuretic effects. Administration of GLP-1 RA increases the fractional excretion of sodium and urine volume $[65,67,69,70]$. Natriuresis induced by GLP-1 RA likely result in large part from blockade of the sodium-hydrogen exchange transporter isoform 3 (NHE3) at the proximal tubule. In vitro, the GLP-1 agonist exendin-4, inhibits NHE3 through modulation of protein kinase A and cyclic adenosine monophosphate, culminating in downstream phosphorylation of the NHE3 transporter without changes in the surface expression within renal tubular cells [71]. This results in natriuretic effects [72] in a variety of animal models [73]. It would perhaps be anticipated that a proximal natriuresis should activate tubuloglomerular feedback at the macula densa, thereby causing afferent arteriolar vasoconstriction and a decrease in GFR. In contrast with this expected effect, eGFR does not change in patients with T2DM in response to GLP-1 RA in response to chronic treatment. First, in pivotal trials of GLP-1 RAs, there is no acute decrease in GFR that would be expected with proximal natriuresis, suggesting that there is minimal renal hemodynamic response to GLP-1 RAs. In the long-term, a meta-analysis of CVOTs of available GLP-1 RAs did not demonstrate a reduction in the incidence of worsening of kidney function (HR, $0.87 ; 95 \%$ CI, 0.73 to 1.03 ). However, to make matters more complex, there was moderate heterogeneity among the included trials, with an $I^{2}$ of $43 \%$. Notably, in the Researching Cardiovascular Events With a Weekly Incretin in Diabetes (REWIND) trial comparing dulaglutide to placebo, there was an $11 \%$ reduction in the incidence of a sustained decline in eGFR of $\geq 30 \%$ (HR, 0.89 ; $95 \%$ CI, 0.78 to 1.01 ) but further sensitivity analyses showed a $30 \%$ and $44 \%$ reduction in the incidence of a sustained decline of eGFR of $\geq 40 \%$ (HR, $0.70 ; 95 \% \mathrm{CI}$, 0.57 to 0.85 ) and $\geq 50 \%$ (HR, $0.56 ; 95 \% \mathrm{CI}, 0.41$ to 0.76 ) respectively [74]. The reason for modest, albeit highly variable, longer-term effects on GFR despite a proximal natriuresis is not known. Given the complex mechanisms at play, it may be that in the overall population of adults with diabetes, GLP-1 RAs have minimal effects on GFR but may be more beneficial within specific subpopulations.

Beyond effects on GFR, GLP-1 RAs reduce albuminuria. For instance, liraglutide and semaglutide were associated with a $26 \%$ reduction (HR, $0.74 ; 95 \%$ CI, 0.60 to 0.91 ) and $46 \%$ reduction (HR, $0.54 ; 95 \% \mathrm{CI}, 0.37$ to 0.77 ) in the incidence of new onset persistent macroalbuminuria compared to placebo in the Liraglutide Effect and Action in Diabetes: Evaluation of Cardiovascular Outcome Results (LEADER) and Trial to Evaluate Cardiovascular and Other Long-term Outcomes With Semaglutide in Subjects With Type 2 Diabetes (SUSTAIN-6) trials, respectively $[75,76]$. Likewise, dulaglutide was associated with a $23 \%$ reduction in new onset macroalbuminuria (HR, $0.77 ; 95 \% \mathrm{CI}, 0.68$ to 0.87 ) compared to placebo in the REWIND trial [74]. A Research Study to See How Semaglutide Works Compared to Placebo in People With Type 2 Diabetes and Chronic Kidney Disease (FLOW, NCT03819153) is currently recruiting, and its primary endpoint is a composite outcome defined as persistent eGFR decline of greater than or equal to $50 \%$, reaching ESKD, death from kidney disease or death from $\mathrm{CV}$ disease.

The lack of a robust or consistent hemodynamic effect with GLP-1 RAs suggest that non-hemodynamic mechanisms could be responsible for the decline in albuminuria and possible eGFR preservation with these agents, including improvements in glucose control and metabolic parameters. Potential non-glycemic factors that may be kidney protective with GLP-1 RA include suppression of inflammatory pathways by preventing macrophage infiltration and decreasing oxidative stress [77], as re- 
ported in experimental models. Finally, reductions in body weight and blood pressure may also be beneficial, although the impact of these modest changes is less likely to be clinically relevant.

\section{GLP-1 RA and effects on cardiovascular risk}

From a CV perspective, GLP-1 RAs reduce CV risk compared to placebo in CVOTs. In a recent meta-analysis including all available CVOTs for GLP-1 RAs, all-cause mortality was reduced by $12 \%$ (HR, $0.88 ; 95 \%$ CI, 0.83 to 0.95$)$, CV mortality by $12 \%$ (HR, $0.88 ; 95 \% \mathrm{CI}, 0.81$ to 0.96$)$ and three-point MACE by $12 \%$ (HR, $0.82 ; 95 \%$ CI, 0.82 to 0.94 ) compared to placebo [78]. While there was moderate statistical heterogeneity for three-point MACE in these analyses, there was no evidence of subgroup differences based on the pharmacologic characteristics of studied agents or the baseline characteristics of adults enrolled in the clinical trials. Importantly, the benefits for MACE were consistent in adults with and without DKD.

Beyond effects on MACE and atherosclerosis, HF is an important source of morbidity and mortality in adults with diabetes. HF is in part characterized by myocardial insulin resistance, which contributes to impaired calcium uptake and mitochondrial function within the heart $[79,80]$. Therefore, agents that alter insulin sensitization or release have been theorized as possible treatments for these metabolic abnormalities seen in the setting of HF. In contrast with SGLT2 inhibitors, GLP-1 RAs have only a modest effect on the risk of HF hospitalization. For instance, in meta-analyses, GLP-1 RAs were associated with a 9\% reduction in the incidence of $\mathrm{HHF}$ (HR, $0.91 ; 95 \% \mathrm{CI}, 0.83$ to 0.99 ) [78]. Likewise, for acute decompensated HF, there was no evidence of benefit with respect to HF re-hospitalization for liraglutide compared with placebo [81]. Therefore, combining GLP-1 RAs with SGLT2 inhibitors in clinical practice may be a strategy to leverage the benefits of each agent for MACE and HF respectively. Further research is needed to better ascertain the overall benefit-risk profile of this approach.

\section{NON-STEROIDAL MINERALOCORTICOID RECEPTOR ANTAGONISTS, CARDIOVASCULAR AND KIDNEY PROTECTION IN PATIENTS WITH T2DM}

\section{The case for additional RAAS blockade in diabetic kidney disease}

RAAS blockade is a pillar of DKD management, with benefits demonstrated in clinical trials for both primary and secondary prevention [82-86]. However, even when used in conjunction with SGLT2 inhibitors, patients with DKD continue to have significant residual risk of progression [46,47]. After success in trials with single agent RAAS blockade, interest shifted to dual RAAS blockade. The Ongoing Telmisartan Alone and in combination with Ramipril Global Endpoint trial (ONTARGET) was one of the earlier trials of dual RAAS blockade, randomizing 25,000 participants with vascular disease or diabetes to ramipril, telmisartan, or both. Dual therapy did reduce proteinuria to a greater extent, albeit in the context of increased rates of kidney function decline, dialysis, or death [87]. The Veteran Affairs-Diabetes in Nephropathy Study (VA-NEPHRON-D) and Aliskiren Trial in Type 2 Diabetes Using Cardiovascular and Renal Disease Endpoints (ALTITUDE) trials similarly assessed combination RAAS blockade but were discontinued early due to increased rates of hyperkalemia, AKI, and hypotension $[88,89]$. A role for mineralocorticoid receptor antagonism in DKD has been appealing with a strong physiological rationale, since mineralocorticoid receptors are widely expressed in kidney and cardiac tissue, and their activation by aldosterone leads to hemodynamic and non-hemodynamic effects culminating in inflammation, fibrosis, and progression of cardiac and kidney disease [90-97]. Until recently, clinical studies of MRA in CKD had not looked at 'hard' outcomes, although there is increased risk of hyperkalemia as demonstrated by a Cochrane review summarizing the literature up to but excluding Efficacy and Safety of Finerenone in Subjects With Type 2 Diabetes Mellitus and Diabetic Kidney Disease (FIDELIO-DKD) [98].

\section{Novel mineralocorticoid receptor antagonists in diabetic kidney disease}

Considering the uncertainty surrounding the use of traditional MRAs in CKD despite the establishment of their therapeutic role in HF with reduced ejection fraction, there has been growing interest in novel MRAs with higher selectivity, potency and with reduced risk of hyperkalemia [99]. FIDELIO-DKD was a phase 3 trial of finerenone, in addition to standard of care, in participants with T2DM, moderately advanced CKD (mean baseline eGFR was $44 \mathrm{~mL} / \mathrm{min} / 1.73 \mathrm{~m}^{2}$ with a median UACR of $852 \mathrm{mg} / \mathrm{g}$ ) with the majority (87\%) having macroalbuminuria [100]. The primary outcome was a composite kidney outcome - kidney failure, sustained $\geq 40 \%$ decline in eGFR, or death from renal causes. A secondary composite kidney outcome identical to the primary outcome but with a $57 \%$ decline in eGFR was also included. Finerenone significantly reduced the incidence of the primary and secondary kidney outcomes by 
$18 \%$ and $24 \%$ respectively, with a number needed to treat of 29 patients for the primary outcome. Breaking down the components of kidney composite outcomes, finerenone reduced the $\geq$ $57 \%$ decline in eGFR outcome by $32 \%$ (95\% CI, 0.55 to 0.82 ), and the kidney failure/ESKD by $13 \%$ (95\% CI, 0.72 to 1.05$)$. Examining the between-group eGFR differences, after considering a finerenone associated acute decline in eGFR of $2.5 \mathrm{~mL} /$ $\mathrm{min} / 1.73 \mathrm{~m}^{2}$ compared to placebo, the finerenone group declined by $2.66 \mathrm{~mL} / \mathrm{min} / 1.73 \mathrm{~m}^{2} /$ year versus a $3.97 \mathrm{~mL} / \mathrm{min} / 1.73$ $\mathrm{m}^{2} /$ year decline in the placebo group, with a crossing of the eGFR curves occurring at approximately 24 months. Finerenone additionally reduced UACR by $31 \%$ versus placebo within the first 4 months (95\% CI, 0.66 to 0.71 ) and onwards, and was associated with small reductions in blood pressure (Table 1).

In keeping with established links between kidney and CV health, finerenone also reduced rates of the $\mathrm{CV}$ outcome compared to placebo, an effect that was apparent within a month of starting the study drug [100]. Results of the ongoing Efficacy and Safety of Finerenone in Subjects With Type 2 Diabetes Mellitus and the Clinical Diagnosis of Diabetic Kidney Disease (FIGARO-DKD, NCT02545049) trial can be expected to shed further light on $\mathrm{CV}$ and $\mathrm{HF}$ outcomes.

\section{Non-steroidal mineralocorticoid receptor antagonists and safety}

FIDELIO-DKD did not show an increase in the anticipated adverse events including AKI, breast hyperplasia, or gynecomastia. While finerenone increased the risk of hyperkalemia, the risk of it leading to study drug discontinuation was low $(2.3 \%$ with finerenone compared to $0.9 \%$ with placebo). Additionally, this rate of study drug discontinuation was numerically lower compared to rates in prior studies of dual RAAS inhibition (ALTITUDE 4.8\% and VA-NEPHRON-D 9.9\%). Finerenone also appears to be safer than steroidal MRAs as demonstrated in the ARTS trial where several doses were compared to spironolactone and placebo [101]. While increases in serum potassium levels were observed compared to placebo, the increase was significantly lower compared to spironolactone at all doses of finerenone.

\section{COMBINED USE OF NOVEL AGENTS FOR THE TREATMENT OF DKD}

SGLT2 inhibitors, GLP-1 receptor agonists and non-steroidal MRAs are expected to be increasingly used together in the setting of DKD on a background of single agent RAAS blockade.
Studies examining the use of these agents together in the setting of clinical trials, real world analyses, or mechanistic trials will be important to characterize the nature of their combined heart and kidney benefits. There is some data on the combined use of SGLT2 inhibitors and GLP-1 receptor agonists. The 28-week, phase three, Evaluate Efficacy and Safety of Exenatide Once Weekly and Dapagliflozin Versus Exenatide and Dapagliflozin Matching Placebo (DURATION-8) trial demonstrated that dual therapy with dapagliflozin and exenatide had additive effects on lowering weight and blood pressure, though with a less than additive effect on HbA1c [102]. Additive effects on weight loss were similarly demonstrated in the Efficacy and Safety of Semaglutide Once-weekly Versus Placebo as add-on to SGLT$2 \mathrm{i}$ in Subjects With Type 2 Diabetes Mellitus (SUSTAIN-9) and Dulaglutide as add-on therapy to SGLT2 inhibitors in patients with inadequately controlled type 2 diabetes (AWARD-10) trials $[103,104]$. The effect of such combination therapy on heart and kidney outcomes in DKD is unknown, though it is conceivable that additive benefits on weight loss and blood pressure would confer additive benefits on harder clinical outcomes (Table 1). A propensity-matched cohort from the Exenatide Study of Cardiovascular Event Lowering Trial (EXSCEL) trial demonstrated that the addition of exenatide in patients on a background of SGLT2 inhibition resulted in modest improvements in MACE, all-cause mortality and slower rates of renal decline compared to patients not on an SGLT2 inhibitor [105]. Results of the FLOW trial (NCT03819153) are also anticipated as a larger proportion of participants will be expected to be on concomitant SGLT2 inhibition. Based on the study design of FIDELIO-DKD, finerenone is intended to be used as add-on therapy to ACE inhibition or ARBs, and only a small proportion of participants were treated with SGLT2 inhibition due to the era of the trial. There additionally exists a safety argument for using MRAs in combination with SGLT2 inhibitors to reduce the risk of hyperkalemia, since the latter medication class has a modest potassium lowering effect as suggested by a recent post hoc analysis of MRA use in DAPA-HF [106]. Only $4 \%$ to $11 \%$ of FIDELIO-DKD participants were on an SGLT2 inhibitor over the course of the study, and CREDENCE did not enroll participants taking an MRA at baseline, so there is limited evidence for the combined use of these two agents currently. See Fig. 1 regarding the possible sequential or combined use of these three classes of medications, starting with the areas of strongest evidence. 


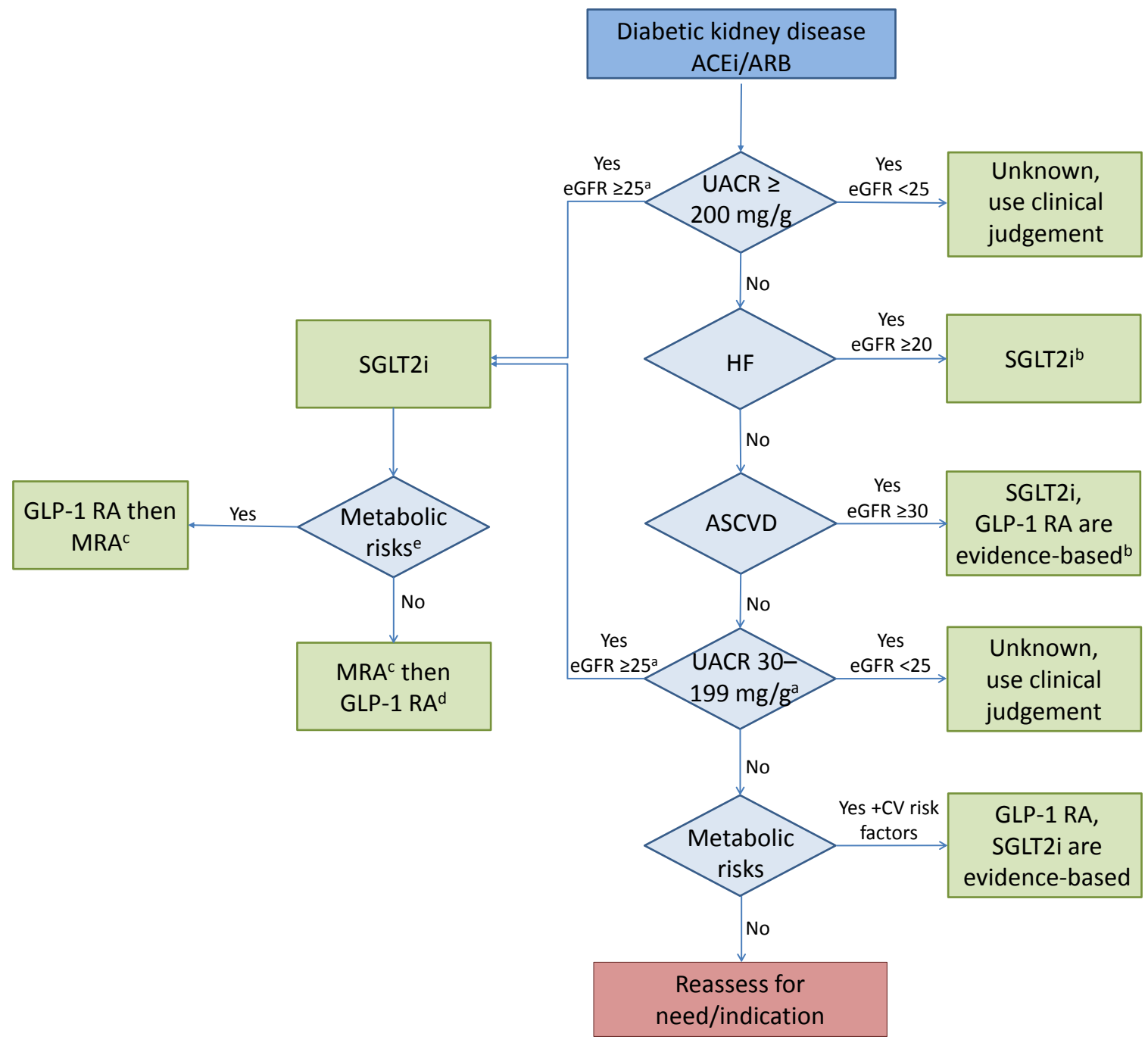

Fig. 1. Decision algorithm for prescribing sodium-glucose cotransporter-2 inhibitor (SGLT2i), glucagon-like peptide-1 receptor agonist (GLP-1 RA) and mineralocorticoid receptor antagonist (MRA) to optimize heart and kidney protection in patients with diabetic kidney disease. ACEi, angiotensin converting enzyme inhibitor; ARB, angiotensin receptor blocker; UACR, urinary albumin-to-creatinine ratio; HF, heart failure; ASCVD, atherosclerotic cardiovascular disease. 'EMPA KIDNEY trial may allow use of SGLT2i down to eGFR of 20 without albuminuria; ${ }^{b}$ FIGARO trial in progress which will give further information on use of MRA in HF and ASCVD; ${ }^{\mathrm{c} A d d}$ on if residual albuminuria with UACR $>30 \mathrm{mg} / \mathrm{g}$ despite optimal medical therapy; ${ }^{\mathrm{d}} \mathrm{FLOW}$ trial may suggest GLP-1 RA use to prevent composite renal out-

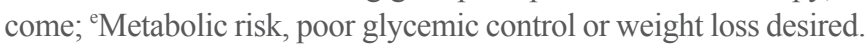

\section{CONCLUSIONS}

The management of DKD has evolved over the last 5 years with a renewed emphasis on therapeutic agents associated with heart and kidney protection. SGLT2 inhibitors have established benefits on kidney, CV and HF outcomes in the setting of DKD. GLP-1 RA therapies have also been noted to have heart and kidney protective effects, although benefits in the kidney at this time appear to be isolated to preventing the progression of proteinuria, and $\mathrm{CV}$ benefits appear to be primarily anti-atheroscle- rotic rather than on the basis of preventing HHF with SGLT2 inhibitors. Finally, non-steroidal MRAs are the latest addition to the DKD armamentarium with evidence of kidney and CV benefits. Further work is required to determine the impact of combinations of these therapies on heart and kidney endpoints in patients with diabetes.

\section{CONFLICTS OF INTEREST}

David Z.I. Cherney has received honoraria from Boehringer In- 
gelheim-Lilly, Merck, AstraZeneca, Sanofi, Mitsubishi-Tanabe, Abbvie, Janssen, Bayer, Prometic, BMS, Maze and Novo-Nordisk and has received operational funding for clinical trials from Boehringer Ingelheim-Lilly, Merck, Janssen, Sanofi, AstraZeneca and Novo-Nordisk. Others have no conflicts of interest to declare.

\section{ACKNOWLEDGMENTS}

Vikas S. Sridhar is supported by the Department of Medicine Eliot Phillipson Clinician Scientist Training Program and a Banting and Best Diabetes Centre Postdoctoral fellowship at the University of Toronto. David Z.I. Cherney is supported by a Department of Medicine, University of Toronto Merit Award and receives support from the CIHR, Diabetes Canada and the Heart and Stroke Richard Lewar Centre of Excellence.

\section{ORCID}

Jason F. Lee https://orcid.org/0000-0002-0254-6432

David Z.I. Cherney https://orcid.org/0000-0003-4164-0429

\section{REFERENCES}

1. Tuttle KR, Cherney DZ; Diabetic Kidney Disease Task Force of the American Society of Nephrology. Sodium glucose cotransporter 2 inhibition heralds a call-to-action for diabetic kidney disease. Clin J Am Soc Nephrol 2020;15: 285-8.

2. Tsapas A, Avgerinos I, Karagiannis T, Malandris K, Manolopoulos A, Andreadis P, et al. Comparative effectiveness of glucose-lowering drugs for type 2 diabetes: a systematic review and network meta-analysis. Ann Intern Med 2020; 173:278-86.

3. Sridhar VS, Rahman HU, Cherney DZI. What have we learned about renal protection from the cardiovascular outcome trials and observational analyses with SGLT2 inhibitors? Diabetes Obes Metab 2020;22 Suppl 1:55-68.

4. Lytvyn Y, Bjornstad P, van Raalte DH, Heerspink HL, Cherney DZI. The new biology of diabetic kidney diseasemechanisms and therapeutic implications. Endocr Rev 2020;41:202-31.

5. Dubrofsky L, Srivastava A, Cherney DZ. Sodium-glucose cotransporter-2 inhibitors in nephrology practice: a narrative review. Can J Kidney Health Dis 2020;7:2054358120935701.

6. Sridhar VS, Dubrofsky L, Boulet J, Cherney DZ. Making a case for the combined use of SGLT2 inhibitors and GLP1 receptor agonists for cardiorenal protection. J Bras Nefrol 2020;42:467-77.

7. van Bommel EJM, Lytvyn Y, Perkins BA, Soleymanlou N, Fagan NM, Koitka-Weber A, et al. Renal hemodynamic effects of sodium-glucose cotransporter 2 inhibitors in hyperfiltering people with type 1 diabetes and people with type 2 diabetes and normal kidney function. Kidney Int 2020;97: 631-5.

8. Cherney DZ, Kanbay M, Lovshin JA. Renal physiology of glucose handling and therapeutic implications. Nephrol Dial Transplant 2020;35(Suppl 1):i3-12.

9. Heerspink HJL, Kosiborod M, Inzucchi SE, Cherney DZI. Renoprotective effects of sodium-glucose cotransporter-2 inhibitors. Kidney Int 2018;94:26-39.

10. Cherney DZI, Dekkers CCJ, Barbour SJ, Cattran D, Abdul Gafor AH, Greasley PJ, et al. Effects of the SGLT2 inhibitor dapagliflozin on proteinuria in non-diabetic patients with chronic kidney disease (DIAMOND): a randomised, double-blind, crossover trial. Lancet Diabetes Endocrinol 2020;8:582-93.

11. Heerspink HJL, Karasik A, Thuresson M, Melzer-Cohen C, Chodick G, Khunti K, et al. Kidney outcomes associated with use of SGLT2 inhibitors in real-world clinical practice (CVD- REAL 3): a multinational observational cohort study. Lancet Diabetes Endocrinol 2020;8:27-35.

12. Cherney DZI, Zinman B, Inzucchi SE, Koitka-Weber A, Mattheus M, von Eynatten M, et al. Effects of empagliflozin on the urinary albumin-to-creatinine ratio in patients with type 2 diabetes and established cardiovascular disease: an exploratory analysis from the EMPA-REG OUTCOME randomised, placebo-controlled trial. Lancet Diabetes Endocrinol 2017;5:610-21.

13. Cherney DZ, Odutayo A, Aronson R, Ezekowitz J, Parker JD. Sodium glucose cotransporter-2 inhibition and cardiorenal protection: JACC review topic of the week. J Am Coll Cardiol 2019;74:2511-24.

14. Lopaschuk GD, Verma S. Mechanisms of cardiovascular benefits of sodium glucose co-transporter 2 (SGLT2) inhibitors: a state-of-the-art review. JACC Basic Transl Sci 2020; 5:632-44.

15. Neuen BL, Cherney DZ, Jardine MJ, Perkovic V. Sodiumglucose cotransporter inhibitors in type 2 diabetes: thinking beyond glucose lowering. CMAJ 2019;191:E1128-35.

16. Heerspink HJ, Perkins BA, Fitchett DH, Husain M, Cherney DZ. Sodium glucose cotransporter 2 inhibitors in the 
treatment of diabetes mellitus: cardiovascular and kidney effects, potential mechanisms, and clinical applications. Circulation 2016;134:752-72.

17. Cherney DZI, Cooper ME, Tikkanen I, Pfarr E, Johansen OE, Woerle HJ, et al. Pooled analysis of Phase III trials indicate contrasting influences of renal function on blood pressure, body weight, and $\mathrm{HbA} 1 \mathrm{c}$ reductions with empagliflozin. Kidney Int 2018;93:231-44.

18. Ferrannini E, Mark M, Mayoux E. CV protection in the EMPA-REG OUTCOME trial: a "thrifty substrate" hypothesis. Diabetes Care 2016;39:1108-14.

19. Rojas-Morales P, Tapia E, Pedraza-Chaverri J. $\beta$-Hydroxybutyrate: a signaling metabolite in starvation response? Cell Signal 2016;28:917-23.

20. Youm YH, Nguyen KY, Grant RW, Goldberg EL, Bodogai M, Kim D, et al. The ketone metabolite $\beta$-hydroxybutyrate blocks NLRP3 inflammasome-mediated inflammatory disease. Nat Med 2015;21:263-9.

21. Osataphan S, Macchi C, Singhal G, Chimene-Weiss J, Sales V, Kozuka C, et al. SGLT2 inhibition reprograms systemic metabolism via FGF21-dependent and -independent mechanisms. JCI Insight 2019;4:e123130.

22. Umino H, Hasegawa K, Minakuchi H, Muraoka H, Kawaguchi T, Kanda T, et al. High basolateral glucose increases sodium-glucose cotransporter 2 and reduces sirtuin-1 in renal tubules through glucose transporter-2 detection. Sci Rep 2018;8:6791.

23. Packer M. Cardioprotective effects of sirtuin-1 and its downstream effectors: potential role in mediating the heart failure benefits of sglt2 (sodium-glucose cotransporter 2) inhibitors. Circ Heart Fail 2020;13:e007197.

24. Hesp AC, Schaub JA, Prasad PV, Vallon V, Laverman GD, Bjornstad $\mathrm{P}$, et al. The role of renal hypoxia in the pathogenesis of diabetic kidney disease: a promising target for newer renoprotective agents including SGLT2 inhibitors? Kidney Int 2020;98:579-89.

25. Burns KD, Cherney D. Renal angiotensinogen and sodiumglucose cotransporter-2 inhibition: insights from experimental diabetic kidney disease. Am J Nephrol 2019;49: 328-30.

26. Cherney D, Perkins BA, Lytvyn Y, Heerspink H, Rodriguez-Ortiz ME, Mischak $\mathrm{H}$. The effect of sodium/glucose cotransporter 2 (SGLT2) inhibition on the urinary proteome. PLoS One 2017;12:e0186910.

27. Cherney DZ, Perkins BA, Soleymanlou N, Xiao F, Zimpelmann J, Woerle HJ, et al. Sodium glucose cotransport-2 in- hibition and intrarenal RAS activity in people with type 1 diabetes. Kidney Int 2014;86:1057-8.

28. Dekkers CCJ, Petrykiv S, Laverman GD, Cherney DZ, Gansevoort RT, Heerspink HJL. Effects of the SGLT-2 inhibitor dapagliflozin on glomerular and tubular injury markers. Diabetes Obes Metab 2018;20:1988-93.

29. Kopecky C, Lytvyn Y, Domenig O, Antlanger M, Kovarik JJ, Kaltenecker CC, et al. Molecular regulation of the reninangiotensin system by sodium-glucose cotransporter 2 inhibition in type 1 diabetes mellitus. Diabetologia 2019;62: 1090-3.

30. Clementi A, Virzi GM, Brocca A, de Cal M, Vescovo G, Granata A, et al. Cardiorenal syndrome type 4: management. Blood Purif 2013;36:200-9.

31. Zinman B, Wanner C, Lachin JM, Fitchett D, Bluhmki E, Hantel S, et al. Empagliflozin, cardiovascular outcomes, and mortality in type 2 diabetes. N Engl J Med 2015;373: 2117-28.

32. Wiviott SD, Raz I, Bonaca MP, Mosenzon O, Kato ET, Cahn A, et al. Dapagliflozin and cardiovascular outcomes in type 2 diabetes. N Engl J Med 2019;380:347-57.

33. Neal B, Perkovic V, Mahaffey KW, de Zeeuw D, Fulcher G, Erondu N, et al. Canagliflozin and cardiovascular and renal events in type 2 diabetes. N Engl J Med 2017;377:644-57.

34. Wanner C, Inzucchi SE, Lachin JM, Fitchett D, von Eynatten M, Mattheus M, et al. Empagliflozin and progression of kidney disease in type 2 diabetes. N Engl J Med 2016;375: 323-34.

35. Neuen BL, Young T, Heerspink HJL, Neal B, Perkovic V, Billot L, et al. SGLT2 inhibitors for the prevention of kidney failure in patients with type 2 diabetes: a systematic review and meta-analysis. Lancet Diabetes Endocrinol 2019; 7:845-54.

36. Zelniker TA, Raz I, Mosenzon O, Dwyer JP, Heerspink HJL, Cahn A, et al. 192 Effect of dapagliflozin on cardiovascular outcomes in patients with type 2 diabetes according to baseline renal function and albuminuria status: insights from DECLARE-TIMI 58. Eur Heart J 2019;40(Suppl 1):ehz747.0052.

37. Cooper ME, Inzucchi SE, Zinman B, Hantel S, von Eynatten M, Wanner C, et al. Glucose control and the effect of empagliflozin on kidney outcomes in type 2 diabetes: an analysis from the EMPA-REG OUTCOME trial. Am J Kidney Dis 2019;74:713-5.

38. Inzucchi SE, Zinman B, Fitchett D, Wanner C, Ferrannini E, Schumacher M, et al. How does empagliflozin reduce car- 
diovascular mortality? Insights from a mediation analysis of the EMPA-REG OUTCOME trial. Diabetes Care 2018; 41:356-63.

39. Li J, Neal B, Perkovic V, de Zeeuw D, Neuen BL, Arnott C, et al. Mediators of the effects of canagliflozin on kidney protection in patients with type 2 diabetes. Kidney Int 2020; 98:769-77.

40. Li J, Woodward M, Perkovic V, Figtree GA, Heerspink HJL, Mahaffey KW, et al. Mediators of the effects of canagliflozin on heart failure in patients with type 2 diabetes. JACC Heart Fail 2020;8:57-66.

41. Cannon CP, Pratley R, Dagogo-Jack S, Mancuso J, Huyck $\mathrm{S}$, Masiukiewicz U, et al. Cardiovascular outcomes with ertugliflozin in type 2 diabetes. N Engl J Med 2020;383: 1425-35.

42. Cherney DZI, Charbonnel B, Cosentino F, Dagogo-Jack S, McGuire DK, Pratley R, et al. Effects of ertugliflozin on kidney composite outcomes, renal function and albuminuria in patients with type 2 diabetes mellitus: an analysis from the randomised VERTIS CV trial. Diabetologia 2021 Mar 4 [Epub]. https://doi.org/10.1007/s00125-021-05407-5.

43. Cherney DZI, McGuire DK, Charbonnel B, Cosentino F, Pratley R, Dagogo-Jack S, et al. Gradient of risk and associations with cardiovascular efficacy of ertugliflozin by measures of kidney function: observations from VERTIS CV. Circulation 2021;143:602-5.

44. Bhatt DL, Szarek M, Pitt B, Cannon CP, Leiter LA, McGuire DK, et al. Sotagliflozin in patients with diabetes and chronic kidney disease. N Engl J Med 2021;384:129-39.

45. Bhatt DL, Szarek M, Steg PG, Cannon CP, Leiter LA, McGuire DK, et al. Sotagliflozin in patients with diabetes and recent worsening heart failure. N Engl J Med 2021;384: 117-28.

46. Perkovic V, Jardine MJ, Neal B, Bompoint S, Heerspink HJL, Charytan DM, et al. Canagliflozin and renal outcomes in type 2 diabetes and nephropathy. N Engl J Med 2019; 380:2295-306.

47. Heerspink HJL, Stefansson BV, Correa-Rotter R, Chertow GM, Greene T, Hou FF, et al. Dapagliflozin in patients with chronic kidney disease. N Engl J Med 2020;383:1436-46.

48. McMurray JJV, Solomon SD, Inzucchi SE, Kober L, Kosiborod MN, Martinez FA, et al. Dapagliflozin in patients with heart failure and reduced ejection fraction. N Engl J Med 2019;381:1995-2008.

49. Anker SD, Butler J, Filippatos G, Khan MS, Marx N, Lam CSP, et al. Effect of empagliflozin on cardiovascular and renal outcomes in patients with heart failure by baseline diabetes status: results from the EMPEROR-reduced trial. Circulation 2021;143:337-49.

50. Packer M, Anker SD, Butler J, Filippatos G, Pocock SJ, Carson P, et al. Cardiovascular and renal outcomes with empagliflozin in heart failure. N Engl J Med 2020;383:141324.

51. Jhund PS, Solomon SD, Docherty KF, Heerspink HJL, Anand IS, Bohm M, et al. Efficacy of dapagliflozin on renal function and outcomes in patients with heart failure with reduced ejection fraction: results of DAPA-HF. Circulation 2021;143:298-309.

52. Jardine MJ, Zhou Z, Mahaffey KW, Oshima M, Agarwal R, Bakris G, et al. Renal, cardiovascular, and safety outcomes of canagliflozin by baseline kidney function: a secondary analysis of the CREDENCE randomized trial. J Am Soc Nephrol 2020;31:1128-39.

53. Zelniker TA, Wiviott SD, Raz I, Im K, Goodrich EL, Bonaca MP, et al. SGLT2 inhibitors for primary and secondary prevention of cardiovascular and renal outcomes in type 2 diabetes: a systematic review and meta-analysis of cardiovascular outcome trials. Lancet 2019;393:31-9.

54. Huang CY, Lee JK. Sodium-glucose co-transporter-2 inhibitors and major adverse limb events: a trial-level meta-analysis including 51713 individuals. Diabetes Obes Metab 2020;22:2348-55.

55. Sridhar VS, Tuttle KR, Cherney DZI. We can finally stop worrying about SGLT2 inhibitors and acute kidney injury. Am J Kidney Dis 2020;76:454-6.

56. van Raalte DH, Cherney DZI. Sodium glucose cotransporter 2 inhibition and renal ischemia: implications for future clinical trials. Kidney Int 2018;94:459-62.

57. Maiorino MI, Chiodini P, Bellastella G, Capuano A, Esposito K, Giugliano D. Insulin and glucagon-like peptide 1 receptor agonist combination therapy in type 2 diabetes: a systematic review and meta-analysis of randomized controlled trials. Diabetes Care 2017;40:614-24.

58. van Can J, Sloth B, Jensen CB, Flint A, Blaak EE, Saris WH. Effects of the once-daily GLP-1 analog liraglutide on gastric emptying, glycemic parameters, appetite and energy metabolism in obese, non-diabetic adults. Int J Obes (Lond) 2014;38:784-93.

59. Astrup A, Carraro R, Finer N, Harper A, Kunesova M, Lean ME, et al. Safety, tolerability and sustained weight loss over 2 years with the once-daily human GLP-1 analog, liraglutide. Int J Obes (Lond) 2012;36:843-54. 
60. van Baar MJB, van Ruiten CC, Muskiet MHA, van Bloemendaal L, IJzerman RG, van Raalte DH. SGLT2 inhibitors in combination therapy: from mechanisms to clinical considerations in type 2 diabetes management. Diabetes Care 2018;41:1543-56.

61. Richards P, Parker HE, Adriaenssens AE, Hodgson JM, Cork SC, Trapp S, et al. Identification and characterization of GLP-1 receptor-expressing cells using a new transgenic mouse model. Diabetes 2014;63:1224-33.

62. Sun F, Wu S, Guo S, Yu K, Yang Z, Li L, et al. Impact of GLP-1 receptor agonists on blood pressure, heart rate and hypertension among patients with type 2 diabetes: a systematic review and network meta-analysis. Diabetes Res Clin Pract 2015;110:26-37.

63. Pyke C, Heller RS, Kirk RK, Orskov C, Reedtz-Runge S, Kaastrup P, et al. GLP-1 receptor localization in monkey and human tissue: novel distribution revealed with extensively validated monoclonal antibody. Endocrinology 2014; 155:1280-90.

64. Schlatter P, Beglinger C, Drewe J, Gutmann H. Glucagonlike peptide 1 receptor expression in primary porcine proximal tubular cells. Regul Pept 2007;141:120-8.

65. Muskiet MH, Tonneijck L, Smits MM, Kramer MH, Diamant M, Joles JA, et al. Acute renal haemodynamic effects of glucagon-like peptide-1 receptor agonist exenatide in healthy overweight men. Diabetes Obes Metab 2016;18: 178-85.

66. Thomson SC, Kashkouli A, Liu ZZ, Singh P. Renal hemodynamic effects of glucagon-like peptide-1 agonist are mediated by nitric oxide but not prostaglandin. Am J Physiol Renal Physiol 2017 ;313:F854-8.

67. Skov J, Dejgaard A, Frokiaer J, Holst JJ, Jonassen T, Rittig $\mathrm{S}$, et al. Glucagon-like peptide-1 (GLP-1): effect on kidney hemodynamics and renin-angiotensin-aldosterone system in healthy men. J Clin Endocrinol Metab 2013;98:E664-71.

68. Gutzwiller JP, Tschopp S, Bock A, Zehnder CE, Huber AR, Kreyenbuehl M, et al. Glucagon-like peptide 1 induces natriuresis in healthy subjects and in insulin-resistant obese men. J Clin Endocrinol Metab 2004;89:3055-61.

69. Skov J, Pedersen M, Holst JJ, Madsen B, Goetze JP, Rittig $\mathrm{S}$, et al. Short-term effects of liraglutide on kidney function and vasoactive hormones in type 2 diabetes: a randomized clinical trial. Diabetes Obes Metab 2016;18:581-9.

70. Tonneijck L, Smits MM, Muskiet MH, Hoekstra T, Kramer $\mathrm{MH}$, Danser AH, et al. Renal effects of DPP-4 inhibitor sitagliptin or GLP-1 receptor agonist liraglutide in over- weight patients with type 2 diabetes: a 12-week, randomized, double-blind, placebo-controlled trial. Diabetes Care 2016;39:2042-50.

71. Pessoa TD, Campos LC, Carraro-Lacroix L, Girardi AC, Malnic G. Functional role of glucose metabolism, osmotic stress, and sodium-glucose cotransporter isoform-mediated transport on $\mathrm{Na}+/ \mathrm{H}+$ exchanger isoform 3 activity in the renal proximal tubule. J Am Soc Nephrol 2014;25:2028-39.

72. Crajoinas RO, Oricchio FT, Pessoa TD, Pacheco BP, Lessa LM, Malnic G, et al. Mechanisms mediating the diuretic and natriuretic actions of the incretin hormone glucagonlike peptide-1. Am J Physiol Renal Physiol 2011;301:F35563.

73. Farah LX, Valentini V, Pessoa TD, Malnic G, McDonough AA, Girardi AC. The physiological role of glucagon-like peptide-1 in the regulation of renal function. Am J Physiol Renal Physiol 2016;310:F123-7.

74. Gerstein HC, Colhoun HM, Dagenais GR, Diaz R, Lakshmanan M, Pais P, et al. Dulaglutide and renal outcomes in type 2 diabetes: an exploratory analysis of the REWIND randomised, placebo-controlled trial. Lancet 2019;394:131-8.

75. Mann JFE, Orsted DD, Brown-Frandsen K, Marso SP, Poulter NR, Rasmussen S, et al. Liraglutide and renal outcomes in type 2 diabetes. N Engl J Med 2017;377:839-48.

76. Marso SP, Bain SC, Consoli A, Eliaschewitz FG, Jodar E, Leiter LA, et al. Semaglutide and cardiovascular outcomes in patients with type 2 diabetes. N Engl J Med 2016;375: 1834-44.

77. Kodera R, Shikata K, Kataoka HU, Takatsuka T, Miyamoto $\mathrm{S}$, Sasaki M, et al. Glucagon-like peptide-1 receptor agonist ameliorates renal injury through its anti-inflammatory action without lowering blood glucose level in a rat model of type 1 diabetes. Diabetologia 2011;54:965-78.

78. Kristensen SL, Rorth R, Jhund PS, Docherty KF, Sattar N, Preiss D, et al. Cardiovascular, mortality, and kidney outcomes with GLP-1 receptor agonists in patients with type 2 diabetes: a systematic review and meta-analysis of cardiovascular outcome trials. Lancet Diabetes Endocrinol 2019; 7:776-85.

79. Aroor AR, Mandavia CH, Sowers JR. Insulin resistance and heart failure: molecular mechanisms. Heart Fail Clin 2012;8:609-17.

80. Nikolaidis LA, Sturzu A, Stolarski C, Elahi D, Shen YT, Shannon RP. The development of myocardial insulin resistance in conscious dogs with advanced dilated cardiomyopathy. Cardiovasc Res 2004;61:297-306. 
81. Margulies KB, Anstrom KJ, Hernandez AF, Redfield MM, Shah MR, Braunwald E, et al. GLP-1 agonist therapy for advanced heart failure with reduced ejection fraction: design and rationale for the functional impact of GLP-1 for heart failure treatment study. Circ Heart Fail 2014;7:673-9.

82. Diabetes Canada Clinical Practice Guidelines Expert Committee, McFarlane P, Cherney D, Gilbert RE, Senior P. Chronic kidney disease in diabetes. Can J Diabetes 2018;42 Suppl 1:S201-9.

83. Ruggenenti P, Perna A, Ganeva M, Ene-Iordache B, Remuzzi G; BENEDICT Study Group. Impact of blood pressure control and angiotensin-converting enzyme inhibitor therapy on new-onset microalbuminuria in type 2 diabetes: a post hoc analysis of the BENEDICT trial. J Am Soc Nephrol 2006; 17:3472-81.

84. Patel A; ADVANCE Collaborative Group, MacMahon S, Chalmers J, Neal B, Woodward M, et al. Effects of a fixed combination of perindopril and indapamide on macrovascular and microvascular outcomes in patients with type 2 diabetes mellitus (the ADVANCE trial): a randomised controlled trial. Lancet 2007;370:829-40.

85. Parving HH, Lehnert H, Brochner-Mortensen J, Gomis R, Andersen S, Arner P, et al. The effect of irbesartan on the development of diabetic nephropathy in patients with type 2 diabetes. N Engl J Med 2001;345:870-8.

86. Lewis EJ, Hunsicker LG, Clarke WR, Berl T, Pohl MA, Lewis JB, et al. Renoprotective effect of the angiotensin-receptor antagonist irbesartan in patients with nephropathy due to type 2 diabetes. N Engl J Med 2001;345:851-60.

87. Mann JF, Schmieder RE, McQueen M, Dyal L, Schumacher H, Pogue J, et al. Renal outcomes with telmisartan, ramipril, or both, in people at high vascular risk (the ONTARGET study): a multicentre, randomised, double-blind, controlled trial. Lancet 2008;372:547-53.

88. Fried LF, Emanuele N, Zhang JH, Brophy M, Conner TA, Duckworth W, et al. Combined angiotensin inhibition for the treatment of diabetic nephropathy. N Engl J Med 2013; 369:1892-903.

89. Parving HH, Brenner BM, McMurray JJ, de Zeeuw D, Haffner SM, Solomon SD, et al. Cardiorenal end points in a trial of aliskiren for type 2 diabetes. N Engl J Med 2012; 367:2204-13.

90. Viengchareun S, Le Menuet D, Martinerie L, Munier M, Pascual-Le Tallec L, Lombes M. The mineralocorticoid receptor: insights into its molecular and (patho)physiological biology. Nucl Recept Signal 2007;5:e012.
91. Belden Z, Deiuliis JA, Dobre M, Rajagopalan S. The role of the mineralocorticoid receptor in inflammation: focus on kidney and vasculature. Am J Nephrol 2017;46:298-314.

92. Jaisser F, Farman N. Emerging roles of the mineralocorticoid receptor in pathology: toward new paradigms in clinical pharmacology. Pharmacol Rev 2016;68:49-75.

93. Nagase M, Fujita T. Role of Rac1-mineralocorticoid-receptor signalling in renal and cardiac disease. Nat Rev Nephrol 2013;9:86-98.

94. Hollenberg NK. Aldosterone in the development and progression of renal injury. Kidney Int 2004;66:1-9.

95. Fullerton MJ, Funder JW. Aldosterone and cardiac fibrosis: in vitro studies. Cardiovasc Res 1994;28:1863-7.

96. Kawarazaki W, Fujita T. The role of aldosterone in obesityrelated hypertension. Am J Hypertens 2016;29:415-23.

97. Wynn TA, Chawla A, Pollard JW. Macrophage biology in development, homeostasis and disease. Nature 2013;496: 445-55.

98. Chung EY, Ruospo M, Natale P, Bolignano D, Navaneethan SD, Palmer SC, et al. Aldosterone antagonists in addition to renin angiotensin system antagonists for preventing the progression of chronic kidney disease. Cochrane Database Syst Rev 2020;10:CD007004.

99. Cherney DZI, Bakris GL. Novel therapies for diabetic kidney disease. Kidney Int Suppl (2011) 2018;8:18-25.

100. Bakris GL, Agarwal R, Anker SD, Pitt B, Ruilope LM, Rossing P, et al. Effect of finerenone on chronic kidney disease outcomes in type 2 diabetes. N Engl J Med 2020;383: 2219-29.

101. Pitt B, Kober L, Ponikowski P, Gheorghiade M, Filippatos G, Krum H, et al. Safety and tolerability of the novel nonsteroidal mineralocorticoid receptor antagonist BAY 948862 in patients with chronic heart failure and mild or moderate chronic kidney disease: a randomized, double-blind trial. Eur Heart J 2013;34:2453-63.

102. Frias JP, Guja C, Hardy E, Ahmed A, Dong F, Ohman P, et al. Exenatide once weekly plus dapagliflozin once daily versus exenatide or dapagliflozin alone in patients with type 2 diabetes inadequately controlled with metformin monotherapy (DURATION-8): a 28 week, multicentre, double-blind, phase 3, randomised controlled trial. Lancet Diabetes Endocrinol 2016;4:1004-16.

103. Ludvik B, Frias JP, Tinahones FJ, Wainstein J, Jiang H, Robertson KE, et al. Dulaglutide as add-on therapy to SGLT2 inhibitors in patients with inadequately controlled type 2 diabetes (AWARD-10): a 24-week, randomised, 
double-blind, placebo-controlled trial. Lancet Diabetes Endocrinol 2018;6:370-81.

104. Zinman B, Bhosekar V, Busch R, Holst I, Ludvik B, Thielke D, et al. Semaglutide once weekly as add-on to SGLT-2 inhibitor therapy in type 2 diabetes (SUSTAIN 9): a randomised, placebo-controlled trial. Lancet Diabetes Endocrinol 2019;7:356-67.

105. Clegg LE, Penland RC, Bachina S, Boulton DW, Thuresson M, Heerspink HJL, et al. Effects of exenatide and open- label SGLT2 inhibitor treatment, given in parallel or sequentially, on mortality and cardiovascular and renal outcomes in type 2 diabetes: insights from the EXSCEL trial. Cardiovasc Diabetol 2019;18:138.

106. Kristensen SL, Docherty KF, Jhund PS, Bengtsson O, Demets DL, Inzucchi SE, et al. Dapagliflozin reduces the risk of hyperkalaemia in patients with heart failure and reduced ejection fraction: a secondary analysis DAPA-HF. Eur Heart J 2020;41(Suppl 2):ehaa946.093. 